\title{
Can oral rinses play a role in preventing transmission of Covid 19 infection?
}

\author{
Niamh Kelly, ${ }^{1}$ Aoife Nic Íomhair ${ }^{1}$ and Gerry McKenna ${ }^{2}$
}

\section{A commentary on}

\section{O' Donnell V, Thomas D, Stanton $R$ et al.}

Potential role of oral rinses targeting the viral lipid envelope in SARSCoV-2 infection. Function 2020; DOI: 10.1093/function/zqaa002.

\begin{abstract}
This narrative review describes the existing body of evidence supporting the potential role of oral rinses in preventing the transmission of SARS-CoV2. Multiple sources were searched for evidence including PubMed, Researchgate and Google Scholar. References that were identified were then investigated further to find additional source material and the original primary research. Academic and clinical experts were consulted for additional input on virology, immunology, lipid biochemistry, microbicides as well as dental and clinical practice. The authors also collaborated with a number of commercial companies to acquire information on the specific formulations of oral rinses, information not available within the public domain. There were no restrictions on language for the search strategy.
\end{abstract}

\section{Commentary}

Emerging evidence has demonstrated the significant role of the salivary glands and the throat as potential reservoirs for virus replication and transmission in early COVID-19 cases. It has been proposed that viral particles which present in the throat undergo shedding and replication, peaking at 5-6 days and declining thereafter. Although existing research is limited on the methods of viral transmission from the oropharynx to the lungs, possible mechanisms include viral shedding, aspiration of necrotic cell debris or direct infection of adjacent cells. As the route of SARS$\mathrm{CoV}-2$ infection is considered to be via respiratory droplets, strategies to reduce the number of infective virus particles in the oral mucous membranes could contribute to a reduced risk of transmission.

Coronaviruses are surrounded by a 'lipid envelope', into which the spike glycoproteins required for infection are inserted. Disruption of the lipid envelope is an established virucidal strategy used to target coronaviruses. Oral rinses that could damage or destroy the lipid envelope may have the potential to reduce the viral load of SARS-CoV-2 in the mouth and oropharynx.

In-vitro research has investigated the impact of ethanol of enveloped viruses. Dilute formulations of ethanol, glycerol and hydrogen peroxide have been shown to prevent enveloped viral

\section{GRADE rating}

\section{Practice point}

- There is currently insufficient high-quality evidence to suggest that oral rinses are effective against SARS-CoV-2. While a number of guidelines have suggested the use of oral rinses as a prophylactic measure, this should not be as an alternative to high quality personal protective equipment (PPE) and rigorous cross infection control.

replication, including coronaviruses. ${ }^{1}$ However these findings do represent biologically relevant conditions found in the mouth and throat. Further evidence from industry-sponsored studies have demonstrated proof-of-concept that mouthwashes containing essential oils with $2127 \%$ ethanol can inactivate enveloped viruses in both in-vitro and in-vivo experiments with the likely mechanism through damage to the lipid envelope. However specific studies have not been undertaken with the SARS-CoV-2 virus.

The potential use of chlorhexidine, a widely used antibacterial and antifungal mouthwash has also been studied. Formulations are often combined with ethanol at lower concentrations and $0.12 \%$ chlorhexidine has been proven to reduce the viral concentration of enveloped viruses in-vitro (66). A recent review of coronavirus literature indicated rinsing with $0.02 \%$ chlorhexidine for ten minutes only weakly inactivated coronavirus strains. ${ }^{2,3}$ However, combining chlorhexidine with ethanol at appropriate concentrations may be a useful strategy to reduce the viral load as this utilises the substantivity of chlorhexidine within the mouth.

Based on limited in vitro and clinical research, $0.23 \%$ povidoneiodine (PVPI) mouthwash has been shown to inactivate SARSCoV, MERs-CoV and H1N1 influenza virus. Preparations of PVPI containing mouthwash are widely available in Asian countries but it is not currently available within the UK. In Japan, the Ministry of Health, Labour and Welfare have endorsed daily gargling of PVP-I containing mouthwash to prevent upper respiratory tract infections. ${ }^{4}$

Hydrogen peroxide has been shown to disrupt lipid membranes through production of oxygen free radicals. A recent systematic review reported that some enveloped viruses, including coronavirus $229 \mathrm{E}$, are inactivated by $0.5 \%$ hydrogen peroxide. ${ }^{5}$ Higher concentrations of hydrogen peroxide ( $>5 \%$ ) can damage hard and soft intraoral tissues but at lower concentrations it is rapidly inactivated by catalase activity in saliva.

A limited amount of research exists demonstrating the potential role of oral rinses in preventing viral transmission. Low quality evidence suggests a potential role for ethanol containing oral rinses including in combination with essential oils and chlorhexidine. While chlorhexidine demonstrates has substantive uses intra-orally, it does not appear to be effective in reducing viral load. Limited evidence also suggests a potential role for povidoneiodine and hydrogen peroxide. While already published research 
illustrates the potentially prophylactic role of oral rinses to prevent viral disease transmission there is a major clinical need for further research specifically focused on SARS-CoV-2 including clinical trials.

Routine dental care in many parts of the world has been significantly restricted due to the COVID-19 pandemic. The causative organism responsible for the global outbreak has been identified as the novel coronavirus SARS-CoV-2 with Wuhan, Hubei Province, China as the centre of the outbreak. ${ }^{6}$ Due to the nature of dental clinics and hospitals there are significant considerations which must be addressed to prevent cross infection between clinicians and patients, particularly where aerosol generating procedures (AGPs) are undertaken. While transmission of COVID-19 appears to be via respiratory droplets, the mouth, salivary glands and throat are potential reservoirs for virus replication and transmission.

The Centres for Disease Control and Prevention (CDC) have long since advocated the benefits of pre-procedural mouthwashes in reducing airborne pathogens of all types before clinical procedures $^{7}$ Given the challenges of delivering dental procedures during this global pandemic, attention has turned to the potential use of oral rinses to reduce and prevent transmission of SARSCoV-2. This paper presents a review of existing evidence on this topic including oral rinses containing ethanol, chlorhexidine, povidone-iodine, hydrogen peroxide and quaternary ammonium compounds.

Although not a systematic review, considerable efforts have been made by the authors to identify a broad range of evidence relevant to this topic. In addition to a literature search, this included seeking expert advice and opinions on issues requiring clarification, as well as collaboration with commercial companies to seek information not available within the public domain. Given the need for rapid production of evidence during a global pandemic this appears to be a sound methodological approach. It could also be argued that this combination of information from across the scientific community is a necessary approach in the face of such an unknown entity with severe and wide-ranging consequences.

Although providing a summary of the background underpinning the review, the authors do not define specific objectives for the review. While comprehensive, the range of topics covered within the paper, along with the structure used to outline information impairs readability and efficient communication of a succinct and easily digested argument. The review is further limited by a lack of quality assessment and critical appraisal of the studies discussed. The inclusion of the protocol employed for the identification of evidence at the beginning of the paper may also have enabled readers to understand the context of the information included in the main text.

Despite logical scientific reasoning behind the theory proposed, a number of factors prohibit a high degree of confidence in incorporating the theoretical evidence described into clinical practice. The majority of the evidence presented is of low quality with research limited to in-vitro studies and extrapolation of findings from other viruses including influenza, HIV and herpes simplex. These limitations are consistently acknowledged by the authors throughout, who emphasise that the information presented should merely be sufficient to warrant further investigation into the potential offered by the concept. The authors do not overstate their findings and present the limited amount of evidence which suggests a potential role for ethanol containing oral rinses perhaps in combination with essential oils. While chlorhexidine demonstrates useful substantivity intra-orally, it does not appear to be effective in reducing viral load. Limited evidence also suggests a potential role for povidone-iodine and hydrogen peroxide, however, little of the work has been tested in clinical trials.

Unfortunately, given that SARS-CoV-2 is a novel pathogen it should not be a surprise that little or no evidence exists on the effect of oral rinses. The authors clearly specify a number of areas in relation to the role of oral hygiene in dampening the transmission of SARS-CoV-2 that need to be addressed, outlining a variety of research strategies to enable this, including epidemiological studies, clinical trials and populationbased interventions. However, given the unparalleled efforts currently being directed towards other research areas, including vaccination, limited resource availability may preclude the undertaking of such research in a timeframe that would prove beneficial in preventing viral transmission during the current pandemic.

\section{Author affiliations}

${ }^{1}$ Dental Core Trainee, School of Dentistry, Belfast Health and Social Care Trust; ${ }^{2}$ Senior Lecturer / Consultant in Restorative Dentistry, Centre for Public Health, Queen's University Belfast

\section{References}

1. Siddharta A, Pfaender S, Vielle N J et al. Virucidal Activity of World Health Organization-Recommended Formulations Against Enveloped Viruses, Including Zika, Ebola, and Emerging Coronaviruses. J Infect Dis 2017; 215: 902-906.

2. Kampf G, Todt D, Pfaender S, Steinmann E. Persistence of coronaviruses on in animate surfaces and their inactivation with biocidal agents. J Hosp Infect 2020; 104: $246-251$.

3. Saknimit M, Inatsuki I, Sugiyama Y, Yagami K. Virucidal efficacy of physico-chemical treatments against coronaviruses and parvoviruses of laboratory animals. Jikken Dobutsu 1988; 37: 341-345.

4. Japan Ministry of Health LaW. Pandemic influenza preparedness action plan of the Japanese Government 2007.

5. Omidbakhsh N, Sattar S A. Broad-spectrum microbicidal activity, toxicologic assessment, and materials compatibility of a new generation of accelerated hydrogen peroxide-based environmental surface disinfectant. Am J Infect Control 2006; 34: 251-257.

6. Meng L, Hua F, Bian Z. Coronavirus Disease 2019 (COVID-19): Emerging and future challenges for dental and oral medicine. J Dent Res 2020; 99: 481-487.

7. Kohn W G, Collins A S, Cleveland J L et al. Guidelines for infection control in dental health-care settings20-03. MMWR Recomm Rep 2003; 52: 1-61.

Evidence-Based Dentistry (2020) 21, 42-43.

https://doi.org/ 10.1038/s41432-020-0099-1 